\title{
MOTIVAÇÃO E ATIVIDADE FÍSICA: OS PROJETOS DE EXTENSÃO NA UNIVERSIDADE (MOTIVAÇÃO E ATIVIDADE FÍSICA)
}

\author{
Bruna Freitas Romero \\ Universidade Estadual de Campinas, Campinas, São Paulo, Brasil \\ Gabriela Kaiser Fullin Castanho \\ Universidade Estadual de Campinas, Campinas, São Paulo, Brasil \\ João Guilherme Cren Chiminazzo \\ Universidade Estadual de Campinas, Campinas, São Paulo, Brasil \\ Júlia Barreira \\ Universidade Estadual de Campinas, Campinas, São Paulo, Brasil \\ Paula Teixeira Fernandes \\ Universidade Estadual de Campinas, Campinas, São Paulo, Brasil
}

\begin{abstract}
Resumo
O objetivo dessa pesquisa foi identificar os principais fatores que motivam alunos dos Projetos de Extensão da Faculdade de Educação Física da Universidade Estadual de Campinas a praticarem atividade física. $\mathrm{O}$ instrumento utilizado foi o Inventário de Motivação à Prática Regular de Atividade Física (IMPRAF-54), que abrange seis dimensões da motivação (Controle do Estresse, Saúde, Sociabilidade, Competitividade, Estética e Prazer). Foram avaliados 173 sujeitos, entre 18 e 40 anos, de ambos os sexos, que praticam as diferentes modalidades oferecidas pelo projeto. Como resultado geral, verificou-se que a Saúde e o Prazer são os principais motivos para a prática da atividade física, seguidos pelo Controle do Estresse e Estética, Sociabilidade e Competitividade. Ressalta-se a importância de pesquisas assim tanto para a área da Educação Física em geral, quanto para aqueles que trabalham diretamente no oferecimento dessas atividades, no sentido de possibilitar a elaboração de estratégias que mantenham os alunos motivados de maneira adequada para a prática de atividade física.
\end{abstract}

Palavras-chave: Atividade física. Motivação. Universidade. Psicologia do Esporte.

\section{Introdução}

Atualmente, a prática de atividade física pela população vem crescendo significativamente, em especial, devido a uma maior preocupação das pessoas com a qualidade de vida. Por ser uma ferramenta não farmacológica para o envelhecimento saudável (CARVALHO, 2014), aspectos como a melhora da condição física e psicológica, o controle do peso, a diminuição dos níveis de ansiedade e estresse e o menor risco a doenças crônico-degenerativas, são cada vez mais difundidas pelos meios de comunicação, o que contribui para a conscientização das pessoas (TAHARA; SCHWARTZ; SILVA, 2003).

Apesar do aumento, a aderência das pessoas em programas de atividade física ainda é reduzida, podendo estar relacionada com questões motivacionais (LEGNANI et al., 2011), que envolvem aspectos diferentes para cada indivíduo e estão ligadas aos objetivos e necessidades de cada um (ARSEGO, 2012). Devido a esta problemática, tem se tornado de extrema 
relevância estudos que focam na motivação da escolha da prática de alguma atividade, podendo essa ser uma das principais causas para a desistência dos alunos (KLAIN et al., 2014).

Diversos autores criaram teorias na tentativa de descobrir o que pode influenciar e definir o surgimento da motivação nas pessoas. Uma das teorias mais utilizadas é a Teoria da Autodeterminação (Self Determination Theory) (DECI; RYAN, 1985), cuja principal ideia é a influência da autonomia no grau de motivação, chamada de motivação autodeterminada. Neste sentido, a motivação pode ser classificada em três tipos (RYAN; DECI, 2000): 1. Motivação intrínseca, determinada por fatores pessoais, sendo esse o nível da motivação mais autônoma (DECI; VANSTEENKISTE, 2004); 2. Motivação extrínseca, que tem como fonte a expectativa por resultados externos ao indivíduo, como alcançar recompensas ou evitar punições; e 3. A motivação, que é o estado em que a pessoa acredita que a ação não terá relevância para sua vida pessoal, não sabendo informar o motivo para a escolha de determinado comportamento (RYAN; DECI, 2000).

Na psicologia do esporte, Samulski (2002) também utiliza os conceitos externo (fatores ambientais) e interno (fatores pessoais) para explicar a motivação durante a escolha e, posteriormente, a manutenção da prática de uma atividade física. Nesse sentido, o conceito de motivação no esporte geralmente é encaixado em uma dessas três possíveis visões: "A visão centrada no traço", no qual o princípio do surgimento da motivação são as características pessoais do aluno, como a personalidade e os objetivos; "A visão centrada na situação", cuja motivação é sustentada por fatores externos à pessoa, como as instalações do local ou o professor; e "A visão interacional", que é a mais difundida e aceita entre os autores, que relaciona as duas primeiras visões e afirma que a causa da motivação depende desses dois conjuntos de fatores, tanto pessoais quanto do meio (WEINBERG; GOULD, 2001).

Com base nas três visões, pode-se classificar as seis dimensões do IMPRAF da seguinte forma: Prazer e Controle do Estresse como "traço", por se tratarem de motivações intrínsecas; Sociabilidade como "situação", pois a motivação origina-se da interação com outras pessoas; Estética, Saúde e Competitividade como "interacional", pois além de existir o aspecto intrínseco, há também um fator externo que influencia no surgimento da motivação.

Nesse sentido, conhecer os benefícios da atividade física é importante. Seja para manter-se saudável, permanecer com um corpo bonito, aliviar o estresse do dia a dia ou simplesmente para sentir-se bem, sempre haverá algo, em meio a tantos objetivos, que impulsiona o indivíduo a escolher exercitar-se, ou seja, um motivo que o faz adquirir tal comportamento. Compreender quais os aspectos psicológicos envolvidos nesta escolha e entender os fatores motivacionais que influenciam nesse engajamento, é de suma relevância para os profissionais da área do esporte, pois cria a oportunidade da melhor qualidade das aulas, com foco nas expectativas do aluno (COSTA et al., 2003).

O fator de impulsão (motivação), além de se diferenciar de pessoa para pessoa, também pode variar sob o aspecto do contexto em que a ação se dá. $O$ contexto universitário se apresenta como o local de realização do presente estudo, mais especificamente os Projetos de Extensão promovidos pela Faculdade de Educação Física da Unicamp (FEF), que são oferecidos em forma de atividades físicas para toda a comunidade universitária e vem crescendo cada vez mais no tocante ao oferecimento de novas modalidades, bem como o número de pessoas interessadas vem ultrapassando a quantidade de vagas oferecidas. A proposta desta pesquisa foi identificar os principais fatores que motivam os alunos, funcionários e docentes da Universidade Estadual de Campinas a praticarem atividade física dentro do próprio contexto universitário e comparar os fatores motivacionais nas diferentes modalidades esportivas oferecidas. 


\section{Metodologia}

\section{Participantes}

A amostra foi composta por 173 sujeitos - estudantes, funcionários e docentes, homens e mulheres, na faixa etária de 18 e 40 anos de idade e alunos de pelo menos um dos projetos de extensão oferecidos pela Faculdade.

$\mathrm{Na}$ presente pesquisa, os entrevistados participaram das seguintes modalidades de extensão, classificadas em sete grupos: Circo/Ginástica (Trapézio, Tecido, Trampolim Acrobático e Ginástica Acrobática), Condicionamento Físico, Dança (Forró, Dança de Salão e Dança do Ventre), Lutas (Capoeira, Taekwondo, Kung-Fu e Judô), Esportes de Raquete (Tênis e Badminton), Natação, Pilates/Yoga.

\section{Instrumentos}

Os instrumentos utilizados foram:

- Ficha de Identificação, na qual coletou informações sobre o perfil dos sujeitos, bem como possibilitou que esses entrevistados respondessem uma questão aberta sobre os motivos que os levaram a praticar determinada atividade física;

- Inventário de Motivação à Prática Regular de Atividade Física (IMPRAF-54), que possui 54 afirmações divididas em nove grupos. Dessa maneira, são avaliadas seis dimensões: Controle do Estresse (ex: diminuir a ansiedade), Saúde (ex: viver mais), Sociabilidade (ex: fazer novos amigos), Competitividade (ex: competir com os outros), Estética (ex: tornar-se atraente) e Prazer (ex: ter a sensação de bem-estar).

O questionário, criado e validado por Balbinotti e Barbosa (2006), tem suas respostas estruturadas na escala tipo Likert, bidirecional graduada em 5 pontos, sendo que as extremidades, considerando uma pontuação crescente, vão de "isto me motiva pouquíssimo" até "isso me motiva muitíssimo", sendo que os entrevistados deveriam completar a afirmação "realizo atividade física para...". Cada dimensão possui oito itens correspondentes e pode obter uma pontuação que varia de oito a 40 pontos.

\section{Procedimentos utilizados}

Após a autorização do coordenador responsável pela Extensão, o estudo foi enviado e aprovado pelo Comitê de Ética em Pesquisa da Universidade Estadual de Campinas (CAAE: 27429214.6.0000.5404), atendendo às exigências de pesquisas relacionadas com seres humanos.

Todos os participantes que aceitaram participar da pesquisa assinaram o Termo de Consentimento Livre e Esclarecido e receberam a Ficha de Identificação e o Inventário de Motivação à Prática Regular de Atividade Física (IMPRAF-54) para preenchimento no mesmo momento. O tempo de resposta foi em média de 10 minutos.

Para a análise estatística, as medidas de posição (média e mediana) e de dispersão (desvio padrão, mínimo e máximo, quartis e amplitude interquartil) foram utilizadas para resumir o banco de dados coletados. A não normalidade das variáveis quantitativas (dimensões do IMPRAF-54) foi verificada através do teste estatístico de Shapiro-Wilk. Para testar as diferenças entre a motivação dos participantes nas seis dimensões do IMPRAF-54 foi aplicado o teste de Kruskalwallis com post-hoc de Dunns. O mesmo teste foi utilizado para avaliar as motivações dos participantes de diferentes modalidades e de diferentes idades. Dividimos os indivíduos em três grupos etários crescentes, cada um composto por 33\% dos participantes da amostra. O coeficiente de correlação de Spearman foi utilizado para testar a relação entre o 
tempo de prática e a motivação dos entrevistados. O nível de significância adotado foi de 0,05. Todas as análises foram realizadas no programa GraphPadPrism 6.0.

\section{Resultados}

Dos 173 participantes da pesquisa, 103 eram do sexo feminino (59,5\%) e 70, do sexo masculino (40,5\%). Essa prevalência manteve-se em quatro dos grupos de modalidades pesquisados (dança, condicionamento físico, ginástica/circo e pilates/yoga), e o sexo masculino predominou nas modalidades de natação, lutas e esportes de raquete.

Em relação à faixa etária, a média geral de idade foi de 24,8 anos $(\mathrm{DP}= \pm 4,3)$. Quando separada entre os gêneros, tivemos a média de 24,4 anos $(\mathrm{DP}= \pm 3,9)$ para o gênero feminino e 25,5 anos ( $\mathrm{DP}= \pm 4,8)$, para o masculino.

Dentre os entrevistados, houve predominância de alunos da Unicamp frequentando os Projetos de Extensão (86,8\%); já os funcionários totalizaram 3,4\% e docentes apenas 1,1\%. Ainda havia a categoria outros, a qual se incluiu ex-alunos e pesquisadores de pós-doutorado $(8,7 \%)$.

Os sete grupos das modalidades foram formados por: 1) Dança (Dança do Ventre, Dança de Salão e Forró), 33 entrevistados; 2) Condicionamento Físico, 29 entrevistados; 3) Lutas (Kung Fu, Judô, Taekwondo e Capoeira), 24 entrevistados; 4) Natação, 23 entrevistados; 5) Ginástica/Circo (Trapézio, Trampolim, Tecido e Ginástica Acrobática), 20 entrevistados; 6) Esportes de Raquete (Tênis e Badminton), 22 entrevistados; 7) Pilates/Yoga, 22 entrevistados.

Ao analisar as respostas dos entrevistados na questão aberta, foi possível agrupar aquelas que mais se assemelhavam, resultando em cinco categorias, conforme ilustra a Tabela 1 .

Tabela 1 - Motivação para a prática de exercício físico

\begin{tabular}{lcc}
\hline \multicolumn{1}{c}{ Motivo da prática } & Frequência & \% \\
\hline Saúde e bem-estar & 90 & 52 \\
Prazer & 31 & 17,9 \\
Aprendizagem de uma nova atividade & 37 & 21,3 \\
Sair da rotina & 13 & 7,5 \\
Sociabilizar & 2 & 1,1 \\
\hline
\end{tabular}

Fonte: Elaboração própria, 2016.

Saúde e bem-estar foram as respostas apresentadas com mais frequência em relação à motivação para a prática de exercício físico.

A partir do instrumento IMPRAF-54 e dos objetivos da pesquisa, procurou-se observar inicialmente a soma total de cada uma das dimensões, para verificar qual a tendência dos fatores motivacionais, presentes no questionário, nos entrevistados (Tabela 2). 
Tabela 2 - Características das motivações apresentadas pelos entrevistados nas seis dimensões do IMPRAF-54.

\begin{tabular}{ccccccccc}
\hline & Média & D.P. & Mínimo & Máximo & Mediana & Quartil & Quartil & p va- \\
& & & & & & $\mathbf{1}$ & $\mathbf{3}$ & lor \\
\hline CE & 26,6 & 7,9 & 8 & 40 & 27 & 21,0 & 32,5 & \\
SA & 32,3 & 6,8 & 8 & 40 & 34 & 29,0 & 38,0 & \\
SO & 20,3 & 7,5 & 8 & 40 & 20 & 15,0 & 26,0 & \\
CO & 11,2 & 5,2 & 8 & 40 & 8 & 8,0 & 14,0 & \\
ES & 26,0 & 9,0 & 8 & 40 & 27 & 19,5 & 33,0 & \\
PR & 32,0 & 6,5 & 8 & 40 & 33 & 28,0 & 37,0 & \\
\hline
\end{tabular}

Fonte: Elaboração própria, 2016.

Verifica-se que as motivações em todas as dimensões apresentaram diferenças estatísticas significativas entre si, com exceção da saúde e prazer, sendo considerados os fatores que mais motivam o grupo pesquisado no tocante a prática de atividade física, seguidos pelas dimensões de estresse e estética, que também não apresentaram diferenças estatísticas. A sociabilidade, seguida pela competitividade, foram os últimos fatores a motivar os entrevistados a se exercitarem.

A seguir realizou-se uma comparação entre os principais fatores que motivam os entrevistados à prática das diferentes atividades físicas (Figura 1).

Figura 1 - Características das motivações, em cada dimensão do IMPRAF-54, dos indivíduos praticantes de sete diferentes atividades físicas

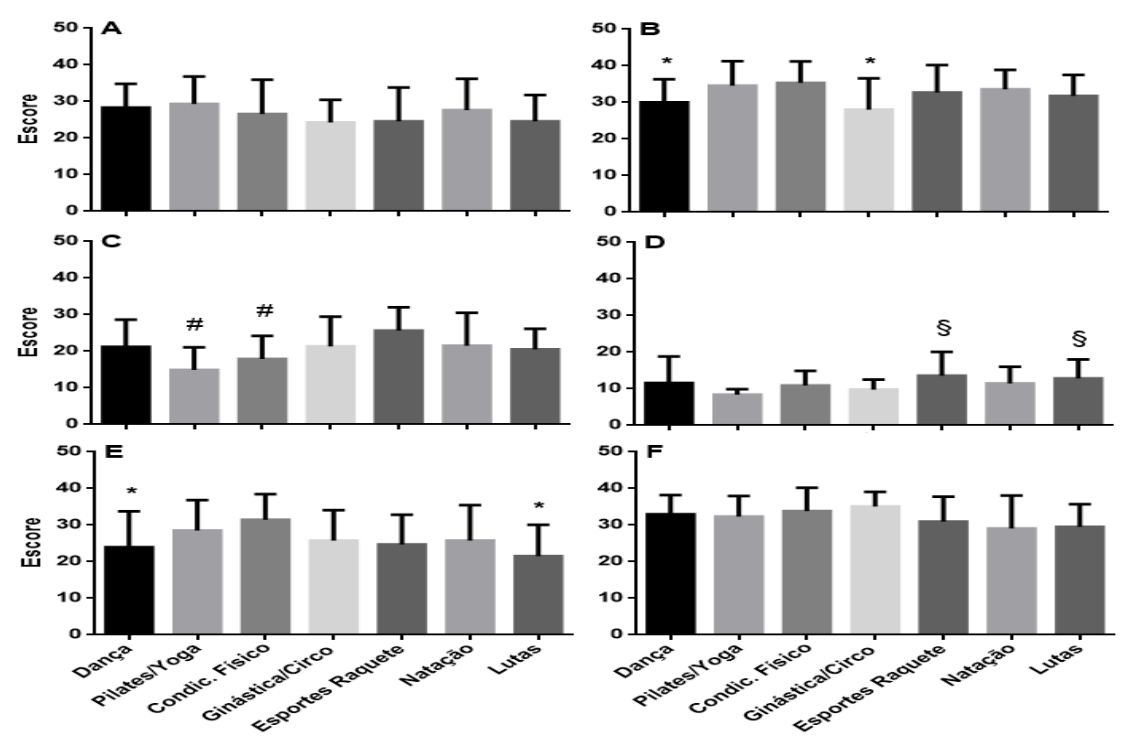

Nota: Dimensões: A) Estresse, B) Saúde, C) Sociabilidade, D) Competitividade, E) Estética, F) Prazer, ${ }^{*}=$ diferença estatisticamente significativa em relação ao condicionamento físico $(\mathrm{p}<0,05), \#=$ diferença estatisticamente significativa em relação aos esportes de raquete 
$(\mathrm{p}<0,05) ; \S=$ diferença estatisticamente significativa em relação ao pilates e yoga $(\mathrm{p}<0,05), \mathrm{p}$ valor $=$ Teste de Kruskalwallis.

Fonte: Elaboração própria, 2016.

Constata-se que, independente da atividade física praticada, os fatores que mais motivam são a saúde e o prazer, e o que menos motiva é a competitividade $(\mathrm{p}<0,05)$.

Verifica-se que os entrevistados apresentaram as mesmas motivações de controle de estresse e de prazer, independente da atividade física realizada. Entretanto, na dimensão da saúde, pode-se observar que os indivíduos que praticam dança ou ginástica/circo se sentem menos motivados em relação aos indivíduos que praticam condicionamento físico. $\mathrm{O}$ aspecto de sociabilidade também apresenta uma menor relevância para os praticantes de pilates/yoga e condicionamento físico em relação aos praticantes de esportes de raquete. $\mathrm{O}$ grupo que prática pilates/yoga também se motiva menos pela competitividade do que os praticantes de esportes de raquete e lutas. E a estética é um fator motivacional maior para os indivíduos que praticam condicionamento físico quando comparados aos que dançam e lutam.

Analisando a motivação em função da idade (Tabela 3), vê-se que os indivíduos com diferentes idades apresentam a mesma motivação à prática esportiva em todas as dimensões, com exceção da saúde. Indivíduos de 27 a 38 anos apresentam níveis de motivação maiores para saúde em relação aos praticantes mais jovens.

Tabela 3 - Motivação dos entrevistados à prática da atividade física em função da idade

\begin{tabular}{|c|c|c|c|c|c|}
\hline & & $\begin{array}{l}18 \text { a } 22 \\
(n=60)\end{array}$ & $\begin{array}{c}23 \text { a } 26 \\
(n=57)\end{array}$ & $\begin{array}{c}27 \text { a } 38 \\
(n=56)\end{array}$ & $P$ valor \\
\hline \multirow{2}{*}{$\mathrm{CE}$} & $\begin{array}{l}\text { Média } \\
\text { (DP) }\end{array}$ & $\begin{array}{l}26,8 \\
(8,4)\end{array}$ & $\begin{array}{l}25,7 \\
(8,1)\end{array}$ & $\begin{array}{l}27,2 \\
(7,3)\end{array}$ & \multirow{2}{*}{0,640} \\
\hline & $\begin{array}{c}\text { Mediana } \\
\text { (Ampl.Inteq) }\end{array}$ & $\begin{array}{c}27,0 \\
(13,0)\end{array}$ & $\begin{array}{c}26,0 \\
(13,0)\end{array}$ & $\begin{array}{c}29,5 \\
(11,0)\end{array}$ & \\
\hline \multirow{2}{*}{ SA } & $\begin{array}{c}\text { Média } \\
\text { (DP) }\end{array}$ & $\begin{array}{l}30,0 \\
(7,8)\end{array}$ & $\begin{array}{l}31,8 \\
(6,4)\end{array}$ & $\begin{array}{c}35,3 * \\
(5,0)\end{array}$ & \multirow{2}{*}{$<0,001$} \\
\hline & $\begin{array}{c}\text { Mediana } \\
\text { (Ampl.Inteq) }\end{array}$ & $\begin{array}{c}31,0 \\
(11,7) \\
\end{array}$ & $\begin{array}{l}33,0 \\
(9,0) \\
\end{array}$ & $\begin{array}{l}37,0 \\
(6,0) \\
\end{array}$ & \\
\hline \multirow{2}{*}{$\mathrm{SO}$} & $\begin{array}{c}\text { Média } \\
\text { (DP) }\end{array}$ & $\begin{array}{l}21,0 \\
(7,9)\end{array}$ & $\begin{array}{l}18,4 \\
(6,8)\end{array}$ & $\begin{array}{l}21,5 \\
(7,6)\end{array}$ & \multirow{2}{*}{0,095} \\
\hline & $\begin{array}{c}\text { Mediana } \\
\text { (Ampl.Inteq) }\end{array}$ & $\begin{array}{c}21,5 \\
(11,2) \\
\end{array}$ & $\begin{array}{c}18,0 \\
(10,5) \\
\end{array}$ & $\begin{array}{c}21,0 \\
(10,7) \\
\end{array}$ & \\
\hline \multirow{2}{*}{$\mathrm{CO}$} & $\begin{array}{l}\text { Média } \\
\text { (DP) }\end{array}$ & $\begin{array}{l}12,0 \\
(6,9)\end{array}$ & $\begin{array}{l}10,4 \\
(3,8)\end{array}$ & $\begin{array}{l}11,3 \\
(4,3)\end{array}$ & \multirow{2}{*}{0,558} \\
\hline & $\begin{array}{c}\text { Mediana } \\
\text { (Ampl.Inteq) }\end{array}$ & $\begin{array}{c}8,0 \\
(5,7) \\
\end{array}$ & $\begin{array}{c}8,0 \\
(3,0) \\
\end{array}$ & $\begin{array}{c}9,0 \\
(7,0) \\
\end{array}$ & \\
\hline \multirow{2}{*}{ ES } & $\begin{array}{l}\text { Média } \\
\text { (DP) }\end{array}$ & $\begin{array}{l}24,3 \\
(9,5)\end{array}$ & $\begin{array}{l}26,8 \\
(8,6)\end{array}$ & $\begin{array}{l}27,0 \\
(8,6)\end{array}$ & \multirow{2}{*}{0,310} \\
\hline & $\begin{array}{c}\text { Mediana } \\
\text { (Ampl.Inteq) }\end{array}$ & $\begin{array}{c}25,0 \\
(18,0) \\
\end{array}$ & $\begin{array}{c}29,0 \\
(11,5) \\
\end{array}$ & $\begin{array}{r}27,0 \\
(13,5) \\
\end{array}$ & \\
\hline PR & $\begin{array}{l}\text { Média } \\
\text { (DP) }\end{array}$ & $\begin{array}{l}32,0 \\
(5,7)\end{array}$ & $\begin{array}{l}31,4 \\
(7,1)\end{array}$ & $\begin{array}{l}32,6 \\
(6,8)\end{array}$ & 0,521 \\
\hline
\end{tabular}




\begin{tabular}{cccc}
\hline Mediana & 32,0 & 33,0 & 33,5 \\
$($ Ampl.Inteq $)$ & $(8,7)$ & $(10,0)$ & $(12,0)$ \\
\hline
\end{tabular}

Nota: $\mathrm{CE}=$ Controle do Estresse; $\mathrm{SA}=$ Saúde; $\mathrm{SO}=$ Sociabilidade; $\mathrm{CO}=$ Competitividade; $\mathrm{ES}=$ Estética; $\mathrm{PR}=$ Prazer, Ampl.Interq $=$ amplitude interquartil, $*=$ diferença estatisticamente significativa em relação aos outros dois grupos etários,p valor $=$ Teste de Kruskalwallis. Fonte: Elaboração própria, 2016.

A Tabela 4 apresenta os resultados da correlação entre o tempo de prática e a motivação nas seis dimensões do IMPRAF-54. Verifica-se que apenas a dimensão de sociabilidade apresentou correlação significativa com o tempo de prática, mostrando que quanto mais tempo os indivíduos praticam as atividades físicas, mais motivados eles são pelo aspecto social da prática. De acordo com as respostas dos entrevistados, 101 iniciaram a prática no mesmo semestre do acontecimento da pesquisa, 19 já praticavam desde o semestre anterior e 53 deles realizavam a atividade física há três semestres ou mais.

Tabela 4 - Correlação entre o tempo de prática na atividade física e a motivação nas seis dimensões do IMPRAF-54

\section{Estresse Saúde Sociabilidade Competitividade Estética Prazer}

$\begin{array}{cccccccc}\begin{array}{c}\text { Tempo } \\ \text { de }\end{array} & \mathrm{R} & 0,13 & 0,09 & 0,25 & 0,12 & 0,04 & 0,10 \\ \text { prática } & \text { Pvalor } & 0,069 & 0,223 & 0,001 & 0,090 & 0,548 & 0,183\end{array}$

Fonte: Elaboração própria, 2016.

\section{Discussão}

A partir dos dados apresentados na Tabela 1, verificamos que, tanto os resultados encontrados nas questões abertas e as respostas obtidas pelo IMPRAF-54, apresentaram semelhança no que diz respeito à primeira e à segunda dimensão mais citada pelos entrevistados: saúde e prazer respectivamente. Contudo, o domínio Saúde e bem-estar foi, sozinho, a resposta que mais ocorreu nas questões abertas, diferentemente do que foi verificado com as respostas do Inventário, no qual Saúde e Prazer não apresentaram diferenças significativas. É possível compreender essa desigualdade considerando que as questões abertas foram aplicadas antes do Inventário, ou seja, não houve nenhuma influência das respostas preestabelecidas por esse instrumento. Além disso, pode-se inferir que o motivo de a maioria das respostas se enquadrarem na categoria Saúde em ambos os questionários é em virtude de, cada vez mais, seja através de pesquisas da área, por prescrição médica ou pelo profissional de educação física, a importância de se praticar atividade física de forma regular tem sido associada à obtenção de um estilo de vida saudável, sendo este um conceito progressivamente absorvido pela maioria da população. Atualmente, na maioria dos casos, os benefícios da prática sob esse aspecto estão relacionados principalmente com a diminuição do risco de doenças crônicas e doenças psicossociais como a ansiedade e a depressão, assim como pode estar associada no tratamento desses quadros, podendo a atividade física atuar positivamente nas dimensões social, emocional, intelectual e física (DANTAS; BEZERRA; MELLO, 2009).

Em relação ao Inventário, em um aspecto geral, o que motiva os participantes desta pesquisa a praticarem atividade física é, em ordem de importância conforme os resultados: 
Saúde e Prazer, Controle do Estresse e Estética, Sociabilidade e, por último, a Competitividade.

De forma semelhante aos resultados encontrados no presente estudo, porém utilizando um questionário desenvolvido e validado no Laboratório de Psicologia e Esporte da Universidade Federal de Minas Gerais, uma pesquisa realizada na UFMG questionou alunos, funcionários e docentes sobre qual a importância de se praticar atividade física. De uma forma geral, os três primeiros motivos indicados, em escala decrescente, foram: Prazer pela atividade física; Melhorar a saúde e Manter-se em forma. Em último na tabela aparecem os motivos Competir com outras pessoas e Aumentar o status social (SAMULSKI; NOCE, 2000). A resposta Redução do estresse do trabalho e Reduzir Stress e Ansiedade aparecem com resultados medianos.

Outra pesquisa (LEGNANI et al., 2011), realizada com estudantes universitários da Universidade Estadual de Londrina, no Paraná, também apontou como principais fatores motivacionais associados à prática de atividade física a Prevenção de doenças e o Prazer e bemestar, seguidos pela Condição Física e Controle do Estresse, sendo a Competição e o Reconhecimento social os fatores menos apontados. Nesse estudo, foi utilizada a versão traduzida e adaptada do instrumento Exercise Motivations Inventory-2 (EMI-2). Mais uma vez, esse resultado se aproxima ao encontrado na população que frequenta os Projetos de Extensão da FEF-UNICAMP.

Na presente pesquisa, em relação às diferentes modalidades, percebe-se que na maioria delas há um padrão das respostas, que se assemelha ao resultado geral. No caso da dimensão competitividade, pode-se notar que, tanto sob o aspecto geral, quanto em cada um dos grupos, esse foi o fator motivacional que menos pontuou. Portanto, pode-se afirmar que essa dimensão não é determinante para a escolha da prática de atividades físicas que fazem parte dos Projetos de Extensão da FEF-UNICAMP.

A sociabilidade mostrou-se como o segundo fator que menos influencia o engajamento na atividade física nesse cenário, exceto no grupo dos esportes de raquete. Analisando a dimensão sociabilidade isoladamente, vê-se que esse fator obteve a maior pontuação nessa modalidade, diferentemente do Condicionamento Físico e do Pilates/Yoga, que tiveram pontuações mais baixas.

A estética e o controle do estresse foram as duas dimensões que se apresentaram com uma influência intermediária em todos os grupos, bem como na análise das médias e pontuações gerais. Nesse sentido, pode-se afirmar que esses dois fatores não são os determinantes principais na decisão de se praticar atividade física por essa população, porém também não apareceram com pontuações baixas como a competitividade e a sociabilidade, representando, dessa forma, um controle regular na decisão dos entrevistados.

As duas dimensões que totalizaram maiores pontuações em todas as modalidades foram a Saúde e o Prazer. Os dois fatores tiveram resultados muito semelhantes, apenas com uma diferença significativa, no qual se percebeu que a dimensão saúde, nas modalidades de dança e ginástica/circo, teve pontuações mais baixas em relação aos outros grupos.

Além dos estudos já citados que também trazem a Saúde e o Prazer como principais fatores motivacionais à prática de atividade física, há também outro estudo (BALBINOTTI et al., 2007) que enfatiza apenas a dimensão Prazer. O objetivo dessa pesquisa foi identificar a variação do nível de influência desse fator durante o período da adolescência em meninos, nas diferentes faixas etárias entre os 13 e 19 anos. Constatou-se que não houve diferenças significativas durante todo o ciclo da adolescência, e que o fator Prazer manteve-se com uma importante influência em relação à prática de atividade física nessa faixa etária. Essa dimensão, que tem sua origem da motivação intrínseca, segundo a Teoria da Autodeterminação, está ligada ao gosto pela prática, sendo que, geralmente, a preferência por determinadas atividades, relacionada ao prazer, são adquiridas durante a infância e adolescência, se mantendo por toda a 
vida. É importante ressaltar que esses resultados podem variar significativamente, tanto em contextos diferentes como em populações de outras faixas etárias. As mesmas modalidades, praticadas em academias ou clubes que possuem o caráter competitivo, por exemplo, poderiam apresentar a dimensão competitividade com pontuações mais elevadas. Como é o caso de uma pesquisa realizada com 166 tenistas da categoria infantojuvenis que participavam de competições e eram filiados às Federações Gaúcha e Catarinense de Tênis (JUCHEM et al., 2007). Os resultados mostraram que o fator competitivo ocupou, junto estatisticamente com a dimensão saúde, o segundo lugar no tocante a motivação para a prática dessa modalidade.

No caso da faixa etária, percebeu-se uma diferença significativa no presente estudo apenas na dimensão saúde nas idades entre 27 e 38 anos, sendo que, nesse grupo, esse aspecto apresentou uma motivação maior. Sobre essa variável, em faixas etárias mais elevadas as diferenças nos resultados podem ser mais visíveis, como mostra um estudo realizado com idosos em um programa de exercícios físicos de Florianópolis, que entrevistou 140 indivíduos através do IMPRAF-54 e obteve como resultado a Saúde, o Prazer e a Sociabilidade como principais fatores motivacionais, além da questão da Recomendação Médica ter sido a resposta que mais apareceu nas questões abertas utilizadas nessa pesquisa (MEURER; BENEDETTI; MAZO, 2012). Já em faixas etárias menores, um estudo realizado na Espanha mostrou que alunos de 12 a 16 anos, dos sexos masculino e feminino, revelam comportamentos diferentes em relação as razões para o interesse pela atividade física. As principais razões que as meninas apresentaram foram o cuidado com a imagem e controle do peso, com o objetivo de evitar problemas e manter a saúde. Enquanto a competição, o desempenho físico e o reconhecimento social englobam as principais razões dos meninos (CASTEDO; PÉREZ, 2015).

No tocante à correlação do tempo de prática e da motivação, verificamos que essa variável não influencia significativamente nas dimensões, sendo que apenas a sociabilidade mostrou uma considerável associação entre os dois fatores. Geralmente, quanto mais tempo os indivíduos praticam a atividade física, mais relações eles estabelecem com os outros praticantes. Por isso, quanto maior o tempo de prática, mais motivados os indivíduos são pelo aspecto social.

Assim, desde períodos mais antigos, já se ressaltava a importância da manutenção de um corpo ativo para a melhora da saúde. Na era atual, na qual há o predomínio das doenças crônico-degenerativas, como a osteoporose, a hipertensão, a diabetes e as dores crônicas, a proporção da ocorrência dessas doenças em indivíduos sedentários acaba sendo muito maior do que nas pessoas que praticam alguma atividade física regularmente (COSTA et al., 2003). Dessa forma, para que o indivíduo mantenha um comportamento ativo, estudos assim tornamse imprescindíveis para que os profissionais da área percebam a importância do seu papel em relação ao fator motivacional e, através de feedbacks e criação de estratégias, possa manter o indivíduo comprometido com um estilo de vida saudável (LIZ et al., 2012).

\section{Conclusão}

De acordo com os resultados deste estudo, a escolha e o engajamento na atividade física nos projetos de extensão FEF-UNICAMP obedecem à seguinte ordem de influência das dimensões (do mais para o menos determinante): Saúde e Prazer, Controle do Estresse e Estética, Sociabilidade e Competitividade. Em relação aos grupos das modalidades, percebeu-se semelhança em comparação com os escores gerais, apresentando algumas diferenças isoladas que foram comentadas.

É importante destacar que, apesar de envolver uma população específica, de forma singular, a pesquisa trouxe informações significativas para os que atuam diretamente na promoção de atividades físicas em contextos universitários, apesar da necessidade de existirem 
mais pesquisas desse âmbito. Dessa forma, a pesquisa possibilita a contribuição da elaboração de um planejamento das aulas que objetivem estimular a motivação dos alunos, de forma a assegurar que a extensão mantenha-se ocupando seu lugar de prestígio dentro da Universidade como um todo.

\title{
MOTIVATION AND PHYSICAL ACTIVITY: EXTENSION PROGRAM AT UNI- VERSITY (MOTIVATION AND PHYSICAL ACTIVITY)
}

\begin{abstract}
The objective of this research was to identify the main factors that motivate students from the Extension Program from Physical Education College at Campinas State University to practice physical activities. The tool used was the Motivational Inventory to the Regular Physical Activity (IMPRAF-54) that covers six dimensions of motivation (Stress Control, Health, Sociability, Competitiveness, Aesthetics and Pleasure). We evaluated 173 subjects, with ages varying from 18-40 years old, who practiced the program's different modalities. Overall, Health and Pleasure were considered the main influence factor to choose the physical activity by the population studied; they were followed by Stress Control, Aesthetics, Sociability and Competitiveness. This study emphasizes the importance of this kind of research to the physical education area and helps who works directly in this kind of activities once it can improve strategies to keep student motivation high when practicing physical activities.
\end{abstract}

Keywords: Physical activities. Motivation. University. Sport psychology.

\section{LA MOTIVACIÓN Y ACTIVIDAD FÍSICA: EL PROYECTO DE EXTENSIÓN DE LA UNIVERSIDAD (MOTIVACIÓN Y ACTIVIDAD FÍSICA)}

\section{Resumen}

El objetivo de esta investigación fue identificar los principales factores que motivan a los estudiantes del Proyecto de Extensión de la Institución Universitaria de Educación Física de la Universidad Estatal de Campinas a practicar actividades físicas. El instrumento utilizado fue el Inventario de Motivación para la Práctica Regular de Actividad Física (IMPRAF-54), que envuelve seis dimensiones de motivación (Control del Estrés, Salud, Sociabilidad, Competitividad, Estética y Placer). Se evaluaron 173 sujetos, de 18 años a 40 años, de ambos sexos, que practican las diferentes modalidades ofrecidas por el proyecto. Como resultado general, se verificó que la Salud y el Placer son los principales motivos para la práctica de la actividad física, seguidos del Control del estrés y la Estética, la Sociabilidad y la Competitividad. Se destaca la importancia de ese tipo de investigación tanto en el ámbito de la educación física en general, como para los que trabajan directamente en la oferta de esas actividades, ya que permite la posibilidad de crear estrategias que mantengan a los estudiantes motivados para la actividad física adecuada. |

Palabras clave: Actividad física. Motivación. Universidad. Psicología del deporte.

\section{Referências}

ARSEGO, N. R. Motivação à prática regular de atividades físicas na academia de ginástica, uma revisão de literatura. 2012. 61 f. Monografia (Bacharelado em Educação Física) Escola de Educação Física, Universidade Federal do Rio Grande do Sul, Porto Alegre, 2012. 
BALBINOTTI, C. A. A et al. A motivação à prática de atividade física regular relacionada ao prazer em adolescentes do sexo masculino. Coleção Pesquisa em Educação Física, v. 6, n. 2, p. 13-18, 2007.

BALBINOTTI, M. A. A.; BARBOSA, M. L. L. Inventário de motivação à prática regular de atividade física (IMPRAF-54). Laboratório de Psicologia do Esporte - Universidade Federal do Rio Grande do Sul, Porto Alegre, 2006.

CASTEDO, A. L.; PÉREZ, M. E. L. Variables motivacionales implicadas en la actividad física en el alumnado de Secundaria. Revista de Estudios e Investigación en Psicología y Educación, v. extr., n. 1, 2015.

CARVALHO, J. Pode o exercício físico ser um bom medicamento para o envelhecimento saudável? Acta Farmacêutica Portuguesa, v. 3, n. 2, p. 123-130, 2014.

COSTA, I. T et al. A importância da atividade física para a manutenção da saúde e os principais fatores que motivam professores, alunos e funcionários de duas universidades brasileiras a praticarem exercícios. Revista Brasileira de Atividade Física \& Saúde, Pelotas, v. 8, n. 1, p. 52-61, 2003.

DANTAS, E. H. M.; BEZERRA, J. C. P.; MELLO, D. B. Fitness Saúde e Qualidade de Vida. In: VIANNA, J.; NOVAES, J. Personal Training \& Condicionamento Físico em Academia. 3. ed. Rio de Janeiro: Shape, 2009. p. 297-335.

DECI, E. L.; RYAN, R. M. Intrinsic Motivation and Self-Determination in Human Behavior. New York: Plenum Press, 1985.

DECI, E. L.; VANSTEENKISTE, M. Self-determination Theory and Basic Need Satisfaction: Understanding human development in positive psychology. Ricerche di Psicologia, v. 27, n. 1, p. 23-40, 2004.

JUCHEM, L et al. A motivação para a prática regular de atividades físicas: um estudo descritivo exploratório com tenistas do sexo masculino de 13 à 16 anos. Coleção Pesquisa em Educação Física, v. 6, n. 2, p. 19-24, 2007.

KLAIN, I. et al. Adesão e desistência de programas de treino personalizado. Revista Iberoamericana de Psicología del Ejercicio y el Deporte, v. 11, n. 1, p. 15-21, 2016.

LEGNANI, R. F. S. et al. Fatores motivacionais associados à prática de exercício físico em estudantes Universitários. Revista Brasileira de Ciências do Esporte, Florianópolis, v. 33, n. 3, p. 761-772, 2011.

LIZ, C. M. et al. O papel do feedback na motivação de praticantes de exercício resistido. Revista Brasileira de Atividade Física e Saúde, v. 17, n. 4, p. 275-278, 2012.

MEURER, S. T.; BENEDETTI, T. R. B.; MAZO, G. Z. Fatores motivacionais de idosos praticantes de exercícios físicos: um estudo baseado na teoria da autodeterminação. Estudos de Psicologia, Natal, v. 17, n. 2, p. 266-303, 2012. 
RYAN, R. M.; DECI, E. L. Intrinsic and extrinsic motivations: Classic definitions and new directions. Contemporary Educational Psychology, v. 25, n. 1, p. 54-67, 2000.

SAMULSKI, D. M.; NOCE, F. A importância da atividade física para a saúde e qualidade de vida: um estudo entre professores, alunos e funcionários da UFMG. Revista Brasileira de Atividade Física \& Saúde, Pelotas, v. 5, n. 1, p. 5-21, 2000.

SAMULSKI, D. Psicologia do Esporte. São Paulo: Manole, 2002.

TAHARA, A. K.; SCHWARTZ, G. M.; SILVA, K. A. Aderência e Manutenção da Prática de Exercícios em Academias. Revista Brasileira de Ciência e Movimento, Brasília, v. 11, n. 4, p. 7-12, 2003.

WEINBERG, R.S.; GOULD, D. Fundamentos da psicologia do esporte e do exercício. 2. ed. Porto Alegre: Artmed, 2001.

Recebido em: 14/11/2016

Revisado em: 02/01/2017

Aprovado em: 23/01/2017

Endereço para correspondência:

paula@fef.unicamp.br

Paula Teixeira Fernandes

Universidade Estadual de Campinas, Faculdade de Educação Física

Avenida Érico Veríssimo, 701

Cidade Universitária

13083-851 - Campinas, SP - Brasil 